\title{
Neuromodulation- an emerging therapeutic modality in neurology
}

Volume I Issue 4 - 2014

Keywords: Neuromodulation, EEG, QEEG, LORETA, Biofeedback, Neurofeedback, Stimulation

\section{Abbreviations: NFB, Neurofeedback; ECT, ElectroconvulsiveTherapy; TMS, Transcranial Magnetic Stimulation; rTMS, repetitive Transcranial Magnetic Stimulation; tDCS, transcranial Direct Current Stimulation; DBS, Deep Brain Stimulation; VNS, Vagus Nerve Stimulation}

Neuromodulation may be defined as the therapeutic alteration of activity in the central, peripheral, or autonomic nervous systems, accomplished by means of self-regulation, stimulation, or medication. The term of neuromodulation is relatively broad and encompasses two main modalities: neuroregulation and neurostimulation.

Neuroregulation represented by Neurofeedback (NFB) also called EEG-biofeedback, has been known as a potential therapeutic modality since 1960's. This type of biofeedback uses real-time displays of EEG to illustrate brain activity and teach self-regulation. Initially, simple systems utilizing one or two EEG sensors were used but many good clinical outcomes required more than 50 sessions. In the 1990s, a new form of EEG biofeedback (Z-score biofeedback) was suggested, in which real-time comparisons to an age matched reference population of healthy or normal subjects were used as a guide to increase specificity and provide a uniform direction and threshold for the biofeedback process.

Prior to $\mathrm{Z}$ score biofeedback, clinicians had to guess about what threshold to set for a given frequency or location to trigger the feedback signal. ${ }^{1} \mathrm{Z}$ score biofeedback greatly simplifies and standardizes EEG biofeedback by reducing many different metrics (absolute power, relative power, ratios, coherence, phase etc.) to a single or common metric of the $\mathrm{Z}$ score or a standard deviation with respect to the EEG from a group. Recent advances in computer technology made this modality more appealing as well as more effective. Especially the full cap EEG-biofeedback systems utilizing 19-active electrodes (which are able to train simultaneously many brain regions) have accelerated the timing of clinical improvement.

Low resolution electromagnetic tomography analysis Neurofeedback (LORETA NFB) added a new three dimensional therapy option based on prior identification of electrical dysregulation in the cerebral cortex. A recently introduced method called LORETA Z-score NFB is capable of targeting specific dysregulated anatomical structures, many of which are in deep cortical locations., ${ }^{2,3}$ For example, the insula and anterior cingulate have been identified as potential NFB target sites for improving pain control in patients who display electrical dysregulation of these areas. ${ }^{4}$ LORETA Z score biofeedback often produces results in one 20 minute session because EEG source localization has accuracies of about $1 \mathrm{~cm}$ to $3 \mathrm{~cm}$ and thus is much more specific than surface EEG. ${ }^{1}$

In the near future, our journal will publish an article with data from my clinic presenting potential effectiveness of LORETA NFB in stroke

\author{
J Lucas Koberda \\ Department of Neurology, Tallahassee NeuroBalance Center, \\ USA
}

Correspondence: J Lucas Koberda, Director of Tallahassee NeuroBalance Center, 4838 Kerry Forest Parkway, Tallahassee, FL 3239, USA, Tel 850-877-2802, Fax 850-222-1383, Email jlkoberda@yahoo.com

Received: September 08, 2014 | Published: September 10, 2014

patients. My center has previously demonstrated clinical efficiency of LORETA Z-score NFB in patients suffering from epilepsy, chronic pain, cognitive dysfunction, Alzheimer's disease, autistic spectrum disorders, attention deficit hyperactivity disorder as well as depression and anxiety. ${ }^{2,4-11}$

Another therapeutic form of neuromodulation is neurostimulation. The oldest form of neurostimulation is known as electroconvulsive therapy (ECT) which is still being used for patients with refractory to medication depression. Newer form of neurostimulation include transcranial magnetic stimulation (TMS) which was initially FDA approved in 2008 for the treatment of individuals with refractory to medications depression. TMS is a noninvasive method to cause depolarization or hyper polarization of the neurons in the brain. TMS uses electromagnetic induction to create weak electric currents using a rapidly changing magnetic field. A variant of TMS called repetitive transcranial magnetic stimulation (rTMS) has been tested as a treatment tool for various neurological and psychiatric disorders including migraine, stroke, Parkinson's disease, dystonia, tinnitus and depression as well as other medical conditions.

Another neurostimulation technique is transcranial direct current stimulation (tDCS) which is still considered as an experimental therapy but it is also gradually gaining grounds in the clinical arena. Tests on healthy adults demonstrated that tDCS can increase cognitive performance on a variety of tasks depending on the area of the brain being stimulated. It has been utilized to enhance language and mathematical ability, attention span, problem solving, memory, and even trading ability.

The modern era of invasive neuromodulation began in the early 1960s, first with deep brain stimulation (DBS) which was soon followed (in 1967) by spinal cord stimulation, both for otherwise intractable pain. DBS is an invasive form of neurostimulation which has been found to be especially effective in therapy for Parkinson's disease and essential tremor. This form of stimulation requires craniotomy and is quite expensive. Vagus nerve stimulation (VNS) was FDA approved in 1997 for the treatment of intractable epilepsy and in 2005 for treatment resistant depression and is less invasive 
than DBS since only a small neck incision is needed for a placement of the stimulator. Most recently in 2014 the FDA has approved a new transcutaneous electrical nerve stimulation device for migraine prevention.

The potential advantage of neuromodulation over pharmacotherapy may have the ability of bypassing the digestive tract and direct (or almost direct) interaction with stimulated neurons. This creates the opportunity to potentially lower side effects while increasing therapeutic gains. Further development of noninvasive neuromodulation techniques will not only be safer for patients but also more cost effective.

\section{Ackowledgments}

None.

\section{Conflicts of interst}

None.

\section{References}

1. Thatcher RW "Handbook of QEEG and EEG Biofeedback". Scientific Foundations and Practical Applications. 2012

2. Koberda JL, Koberda P, Bienkiewicz A, et al. Pain management using 19-electrode Z-score LORETA neurofeedback. Journal of Neurotherapy. 2013;17(3):179-190.

3. Thatcher RW Latest developments in live $\mathrm{z}$-score training: symptom checklist, phase reset, and LORETA z-score Biofeedback. Journal of Neurotherapy. 2013;17(1):69-87.
4. Koberda JL, Koberda L, Koberda P, et al.x Alzheimer's dementia as a potential target of Z-score LORETA 19-electrode Neurofeedback. In: Neuroconnection. Winter Edition, p. 2013;30-32.

5. Koberda JL Autistic Spectrum Disorder (ASD) as a potential target of Z-score LORETA neurofeedback. In: Neuroconnection. ISNR, Winter 2012, USA, p. 2012;24-25.

6. Koberda JL, Moses A, Koberda L, et al. Cognitive enhancement using 19-electrode Z-score neurofeedback. Journal of Neurotherapy. 2012;16(3):224-230.

7. Koberda JL, Koberda P, Moses A, et al. Z-score LORETA Neurofeedback as a potential therapy of ADHD. Summer-Special Edition-Biofeedback Magazine. 2014;42(2):74-81.

8. Koberda JL, Moses A, Koberda P, et al. Cognitive Enhancement with LORETA Z-score Neurofeedback. AAPB meeting. 2014

9. Koberda JL, Moses A, Koberda P, et al. Z-score LORETA neurofeedback as a potential therapy in depression/anxiety and cognitive dysfunction. In: Neuroconnection Spring, USA, p. 2014;52-55.

10. Koberda, JL Therapy of Seizures and Epilepsy with Z-score LORETA Neurofeedback. In: RW Thatcher and JF Lubar (1st edn), Z-score Neurofeedback: Clinical Applications. Academic Press, San Diego, CA (in press). 2014

11. Koberda JL, Stodolska-Koberda U Z-score LORETA neurofeedback as a potential rehabilitation modality in patients with CVA. $J$ Neurol Stroke. 2014 